PROCEEDINGS OF THE

AMERICAN MATHEMATICAL SOCIETY

Volume 133, Number 9, Pages 2733-2735

S 0002-9939(05)08070-6

Article electronically published on April 12, 2005

\title{
A ROUND BALL UNIQUELY MINIMIZES GRAVITATIONAL POTENTIAL ENERGY
}

FRANK MORGAN

(Communicated by Wolfgang Ziller)

\begin{abstract}
We give a proof following Carleman that among measurable bodies in $\mathbf{R}^{3}$ of mass $m_{0}$ and density at most 1 , a round ball of unit density uniquely minimizes gravitational potential energy.
\end{abstract}

\section{INTRODUCTION}

That a round ball uniquely minimizes gravitational potential energy (our Corollary 2.3) was proved by T. Carleman [C] (following Blaschke [B]) in a 1919 German paper in the third volume of Mathematische Zeitschrift. Our main Theorem 2.2 allows variable density and proves that the ball is the unique equilibrium. Our proof (like the Carleman and Blaschke proofs) uses a kind of Steiner symmetrization. By allowing variable density, we can quote standard compactness results, while Carleman uses infinitely many symmetrizations to obtain the ball in the limit.

Earlier, ingenious, physical arguments, relating energy and electric capacitance (Poincaré $[\mathrm{P}$, pp. 13-24] credits Liapounoff), made assumptions about existence and regularity.

Since the time of Newton and before, scientists have studied smooth equilibrium shapes for rotating bodies, sometimes assuming that the body is rigid or incompressible (see e.g. Chandrasekhar [Ch]). Amusingly, there is no minimum for rotating bodies:

Corollary 2.4. Among bodies of mass $m_{0}$, density at most $\delta_{0}$, and angular momentum $L_{0}$ in $\mathbb{R}^{3}$, the infimum of gravitational potential energy plus kinetic energy is approached by a round ball of density $\delta_{0}$ with a small, very distant satellite, but never realized.

\section{ROUND BALLS MINIMIZE GRAVITATIONAL ENERGY}

Before proving our main Theorem 2.2 and Corollaries 2.3 and 2.4 we begin with a slicing characterization of the unit ball.

2.1. Lemma. Let $A \subset \mathbf{R}^{n}$ have unit volume. Suppose that in every direction there is a hyperplane $H$ such that almost every perpendicular line slices $A$ in an interval centered on $H$ (up to measure 0 ). Then $A$ is a round ball (up to measure 0 ).

Received by the editors January 14, 2002.

2000 Mathematics Subject Classification. Primary 76U05, 49Q10, 85A30, 53C80.

Key words and phrases. Rotating fluids, rigid body, gravitational potential energy.

(C)2005 by Frank Morgan 
Proof. First we show that the hyperplanes of symmetry all meet at a point, say the origin. (If $A$ had a well-defined center of mass, for example if $A$ were bounded, we could just use the center of mass.) We may assume that $A$ is invariant under reflection across the axis hyperplanes, hence under $180^{\circ}$ rotation in the $e_{1}, e_{2}$-plane. Hence every hyperplane through the origin with normal in the $e_{1}, e_{2}$-plane splits $A$ in half and must be the hyperplane of symmetry. Consider a hyperplane $H_{3}$ through 0 with normal $n$ in the $e_{1}, e_{2}, e_{3}$-plane; $n$ is a linear combination of $e_{3}$ and a vector $v$ in the $e_{1}, e_{2}$-plane. As before, $A$ is invariant (modulo a set of measure 0 ) under rotation in the $e_{3}, v$-plane, $H_{3}$ splits $A$ in half, and $H_{3}$ must be the hyperplane of symmetry. Proceeding inductively, we conclude that every hyperplane through the origin is a hyperplane of symmetry.

Now for almost every

$$
(p, P) \in A \times\{\text { hyperplanes through the origin }\},
$$

the reflection of $p$ across $P$ lies in $A$. Consequently, almost every point in almost every hypersphere about the origin lies in $A$ or its complement, and (after altering $A$ by a set of measure 0) $A$ is invariant under $S O(n)$. Since almost every linear slice of $A$ is an interval, $A$ is a round ball (up to a set of measure 0 ).

The following theorem is the main result of this paper.

2.2. Theorem. Let $f(t)$ be a strictly decreasing, positive function defined for all $t>0$, with $f(|x-y|)$ locally integrable in $R^{n} \times R^{n}$. Then for measurable density $0 \leq \delta(x) \leq 1$ and unit mass, the energy

$$
E=\iint f(|x-y|) \delta(x) \delta(y) d x d y
$$

is maximum for a round ball of unit density, uniquely up to sets of measure 0. For any other such mass distribution, there is a variation, continuous in time, which increases energy to first order.

Proof. We begin by describing the energy-increasing variation. (This will leave the ball as the only possibility for a maximum, but since we do not yet know that a maximum exists, we will still need to prove the ball maximum.)

So consider any other such mass distribution $\delta(x)$. Unless $\delta(x) \in\{0,1\}$ almost everywhere, choose a hyperplane $H$ so that $\delta(x) \in\{0,1\}$ almost everywhere on neither side of $H$. If $\delta(x) \in\{0,1\}$ almost everywhere, i.e., if $\delta$ is essentially the characteristic function of a set $A$, then by Lemma 2.1 there is a direction with no hyperplane such that almost every slice by a perpendicular line is an interval centered on the hyperplane (up to measure 0). If almost every slice is an interval (up to measure 0), we may choose $H$ such that nontrivial sets of slices have centers above and below $H$. Otherwise we may choose $H$ such that a nontrivial set of slices fail to be intervals (up to measure 0 ) above $H$ and a nontrivial set of slices fail to be intervals (up to measure 0 ) below $H$.

For convenience we may assume that $H$ is the horizontal axis hyperplane, through the origin of every vertical line $L$. Let $I$ be a maximal closed interval in $L$ containing the origin with $\delta(x)=1$ at almost all points, possibly just $\{0\}$. Move mass above $I$ downward with unit velocity. Move mass below $I$ upward with unit velocity. Move $I$ downward or upward at unit velocity (or not at all) towards centering it. Since the density is initially less than 1 on any interval above or below $I$, this flow does not increase density above 1 . If for example $I$ moves upward and there is some mass 
immediately above $I$, then after a small time increment $\Delta t$, an interval immediately above $I$ of length $s+2 \Delta t$ and mass $s$ has become an interval of length $s$, mass $s$, and density 1 , and part of $I$. The mass at time $t$ of an interval about the origin satisfies

$$
m_{t}[-z, z]=\max \left\{2 z, m_{0}[-z-t, z+t]\right\} .
$$

In particular, the mass flows toward a centered interval.

Consideration of the pairwise contribution to energy from the three pieces of any pair of vertical lines shows that energy increases to first order under this flow, as desired.

For use in the next paragraph, we describe a modified flow for densities $\delta(x)$ supported inside a closed ball about the origin. Replace $H$ by its translate through the origin (without translating $\delta$ as we did for convenience before). Now $\delta$ remains supported inside the ball and eventually energy increases.

To prove that the round ball is an energy maximum, it suffices to prove it inside a large ball $B$ about the origin. By weak* compactness of $L^{\infty}(B \times B)$, applied to functions of the form $\delta(x) \delta(y)$ with $\delta$ in $L^{\infty}(B)$ with $0 \leq \delta \leq 1$ and $\int \delta=1$, there is such a $\delta$ which maximizes energy. By the previous paragraph, $\delta$ must be the characteristic function of a round ball of density 1 (up to measure 0 ).

2.3. Corollary. Among bodies of mass $m_{0}$ and density at most $\delta_{0}$ in $\mathbf{R}^{3}$, a round ball of density $\delta_{0}$ has the least gravitational potential energy, uniquely up to sets of measure 0 .

Proof. We may assume that $m_{0}=\delta_{0}=1$. Apply Theorem 2.2 with $f(t)=-G / t$, with $G$ the gravitational constant.

We have the following corollary for rotating bodies [M]:

2.4. Corollary. Among bodies of mass $m_{0}$, density at most $\delta_{0}$, and angular momentum $L_{0}$ in $\mathbb{R}^{3}$, the infimum of gravitational potential energy plus kinetic energy is approached by a round ball of density $\delta_{0}$ with a small, very distant satellite, but never realized.

\section{ACKNOWLEDGMENTS}

I thank Michael Hutchings, Marek Demianski, Ira Wasserman, Janine Wittwer, Bill Wootters, and the referees for the Journal of Mathematical Physics and Mathematics Magazine for their help and inspiration. This work was partially supported by a National Science Foundation grant.

\section{REFERENCES}

[B] Wilhelm Blaschke, Eine isoperimetrische Eigenschaft des Kreises, Math. Z. 1 (1918), 52-57.

[C] T. Carleman, Über eine isoperimetrische Aufgabe und ihre physikalischen Anwendungen, Math. Z. 3 (1919), 1-7.

[Ch] S. Chandrasekhar, Ellipsoidal figures of equilibrium-an historical account, Comm. Pure Appl. Math. 20 (1967), 251-265. MR0213075 (35:3940)

[M] Frank Morgan, The perfect shape for a rotating rigid body, Math. Magazine $\mathbf{7 5}$ (February, 2002), 30-32.

[P] H. Poincaré, Figures d'Equilibre d'une Masse, Paris, Gauthier-Villars, 1902.

Department of Mathematics and Statistics, Williams College, Williamstown, MasSACHUSETTS 01267

E-mail address: Frank.Morgan@williams.edu 